References:

[1] Mease PJ, et al. ACR Open Rheum.2020 Jan;2(1):18-25.

Disclosure of Interests: MARIA MARTIN LOPEZ: None declared, Beatriz Joven-Ibáñez Speakers bureau: Abbvie, Celgene, Janssen, Merck Sharp \& Dohme, Novartis, Pfizer, José Luis Pablos: None declared

DOI: 10.1136/annrheumdis-2020-eular.6579

\section{SAT0429 SECUKINUMAB IMPROVES CLINICAL AND PATIENT-REPORTED OUTCOMES AT 6 MONTHS AMONG PATIENTS WITH PSORIATIC ARTHRITIS IN THE US-BASED CORRONA PSORIATIC ARTHRITIS/SPONDYLOARTHRITIS (PSA/SPA) REGISTRY}

P. J. Mease ${ }^{1}$, T. Blachley ${ }^{2}$, M. Glynn ${ }^{2}$, B. Dube ${ }^{2}$, R. Mclean ${ }^{2}$, N. Kim ${ }^{3,4}$, P. Hur ${ }^{5}$, A. Ogdie ${ }^{6}{ }^{1}$ Swedish Medical Center/Providence St. Joseph Health and University of Washington, Seattle, United States of America; ${ }^{2}$ Corrona, LLC, Waltham, United States of America; ${ }^{3}$ Baylor Scott and White Health, Temple, United States of America; ${ }^{4}$ The University of Texas at Austin, Austin, United States of America; ${ }^{5}$ Novartis Pharmaceuticals Corporation, East Hanover, United States of America; ${ }^{6}$ University of Pennsylvania School of Medicine, Philade/phia, United States of America

Background: Secukinumab, an interleukin-17 antagonist approved for the treatment of PsA, improves all PsA manifestations in the GRAPPA-OMERACT core domain set. ${ }^{1}$ Few US-based studies have evaluated the real-world effectiveness of secukinumab in patients with PsA.

Objectives: To examine clinical and patient-reported outcomes (PROs) in patients with PsA enrolled in the Corrona PsA/SpA registry initiating secukinumab with $\geq 1$ follow-up visit.

Methods: Included were adult patients with PsA in the Corrona registry who initiated secukinumab after April 1, 2017 and remained on secukinumab at their 6-month (window, 5-8 months) follow-up visit. The primary outcome was achievement of minimal disease activity (MDA) at 6 months among patients not in MDA at secukinumab initiation. MDA was defined as meeting 5 of the 7 following criteria: tender joint count (TJC) $\leq 1$, swollen joint count $(\mathrm{SJC}) \leq 1$, psoriasis affected body surface area $(\mathrm{BSA})<3 \%$, patient assessment of pain on visual analog scale (VAS) $\leq 15$, patient global assessment VAS $\leq 20, \mathrm{HAQ}-\mathrm{DI} \leq 0.5$, and tender entheseal points $\leq 1$ using the Leeds Enthesitis Index (LEI). Secondary outcomes included the proportion of patients who achieved resolution (0 sites) of TJC, SJC, enthesitis (using the LEI), and dactylitis among those with $\geq 1$ site at initiation and improvement from baseline in clinical outcomes (BSA, nail psoriasis, physician global assessment, TJC, SJC, and DAPSA) and PROs (patient-reported pain, patient global assessment, HAQ-DI, and Work Productivity and Activity Impairment questionnaire) at 6 months. Outcomes were evaluated in the overall population and in potentially recalcitrant patients with failure of or intolerance to $\geq$ 3 previous biologics to examine if the later line biologic could be adequately effective.

Results: A total of 100 patients with PsA who initiated and maintained secukinumab after 6 months were included. The mean (SD) age was 51.6 (11.6) years, $54.3 \%$ were male, and $96.8 \%$ were white. The mean (SD) symptom and disease duration were $10.8(9.7)$ and $7.0(7.0)$ years, respectively. Thirty patients $(30.0 \%)$ initiated secukinumab $150 \mathrm{mg}$ and $70(70.0 \%)$ initiated secukinumab $300 \mathrm{mg}$. Most (83.0\%) were biologic experienced; 17 patients initiated secukinumab as a 1 st biologic, 34 as $2 n d, 26$ as 3 rd, and 23 as $\geq 4$ th. At initiation, 75/90 patients (83.3\%) were not in MDA; $26 / 71$ (36.6\%) of those with follow-up data available achieved MDA at 6 months (Figure 1). In the overall population, 28 patients $(41.2 \%)$ with TJC $\geq 1,24$ (44.4\%) with SJC $\geq 1,17$ (60.7\%) with enthesitis, and $9(75.0 \%)$ with dactylitis at initiation achieved resolution at 6 months (Table 1). Improvement was observed at 6 months in clinical outcomes and PROs in the overall population (Figures 1 and 2 ) and in patients who initiated secukinumab as $\mathrm{a} \geq 4$ th-line biologic.

Table 1. Resolution of Peripheral Arthritis, Enthesitis, and Dactylitis at 6 Months Among Patients With $\geq 1$ Site at Initiation

\begin{tabular}{lcc}
\hline Secondary Outcomes & $\begin{array}{c}\text { Initiation, } \\
\text { Mean (SD) [n] }\end{array}$ & $\begin{array}{c}\text { 6-Month Follow-Up, } \\
\text { Resolution (Count = 0), } \mathbf{n}(\%)\end{array}$ \\
\hline TJC (1-68) & $9.0(9.7)[68]$ & $28(41.2)$ \\
SJC (1-66) & $4.7(4.2)[54]$ & $24(44.4)$ \\
Enthesitis (1-6) & $1.9(1.1)[28]$ & $17(60.7)$ \\
Dactylitis (1-20) & $2.1(1.3)[12]$ & $9(75.0)$ \\
\hline
\end{tabular}
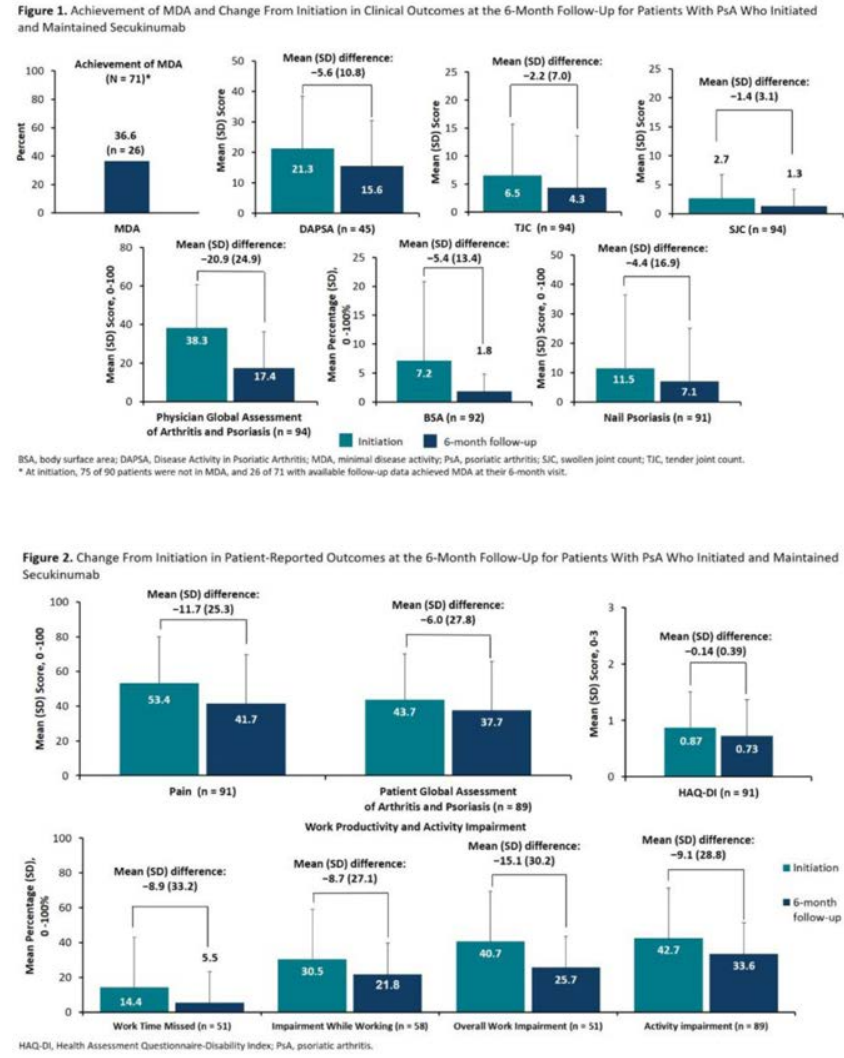

Conclusion: In the Corrona registry, most secukinumab initiators with PsA were biologic experienced and were not in MDA at time of initiation. Consistent with clinical trials, real-world patients treated with secukinumab achieved MDA as well as improvement in clinical manifestations, PROs, and work productivity. References:

[1] Orbai AM, et al. J Rheumatol. 2019 Oct 15. [Epub ahead of print]. Disclosure of Interests: Philip J Mease Grant/research support from: Abbott, Amgen, Biogen Idec, BMS, Celgene Corporation, Eli Lilly, Novartis, Pfizer, Sun Pharmaceutical, UCB - grant/research support, Consultant of: Abbott, Amgen, Biogen Idec, BMS, Celgene Corporation, Eli Lilly, Novartis, Pfizer, Sun Pharmaceutical, UCB - consultant, Speakers bureau: Abbott, Amgen, Biogen Idec, BMS, Eli Lilly, Genentech, Janssen, Pfizer, UCB - speakers bureau, Taylor Blachley Employee of: Corrona, LLC, Meghan Glynn Shareholder of: Corrona, LLC - shareholder, Grant/research support from: Pfizer - grant/research support, Employee of: Corrona, LLC - employment, Blessing Dube Employee of: Corrona, LLC, Robert McLean Employee of: Corrona, LLC, Nina Kim Employee of: Postdoctoral fellow at the University of Texas at Austin and Baylor Scott and White Health, providing services to Novartis Pharmaceuticals Corporation, Peter Hur Employee of: Novartis Pharmaceuticals Corporation, Alexis Ogdie Grant/ research support from: Pfizer to Penn, Novartis to Penn, Amgen to Forward/NDB Consultant of: Abbvie, Amgen, Bristol-Myers Squibb, Celgene, Corrona, Janssen, Eli Lilly, Novartis, Pfizer

DOI: 10.1136/annrheumdis-2020-eular.1014

\begin{tabular}{l|l}
\hline SAT0430 & SECUKINUMAB EFFECTIVENESS IN 1543 PATIENTS \\
WITH PSORIATIC ARTHRITIS TREATED IN ROUTINE \\
CLINICAL PRACTICE IN 13 EUROPEAN COUNTRIES \\
IN THE EUROSPA RESEARCH COLLABORATION \\
NETWORK
\end{tabular}

B. Michelsen ${ }^{1}$, S. Georgiadis ${ }^{1}$, D. DI Giuseppe ${ }^{1}$, A. G. Loft ${ }^{1}$, M. Nissen ${ }^{1}$, F. lannone ${ }^{1}$, M. Pombo-Suarez ${ }^{1}$, H. Mann ${ }^{1}$, Z. Rotar ${ }^{1}$, K. Eklund ${ }^{1}$, T. K. Kvien ${ }^{1}$ M. J. Santos ${ }^{1}$, B. Gudbjornsson ${ }^{1}$, C. Codreanu ${ }^{1}$, S. Yılmaz ${ }^{1}$, J. K. Wallman ${ }^{1}$, C. H. Brahe ${ }^{1}$, B. Moeller ${ }^{1}$, E. G. Favalli ${ }^{1}$, C. Sánchez-Piedra ${ }^{1}$, L. Nekvindova ${ }^{1}$, M. Tomsic ${ }^{1}$, N. Trokovic ${ }^{1}$, E. Kristianslund ${ }^{1}$, H. Santos ${ }^{1}$, T. Love ${ }^{1}$, R. Ionescu ${ }^{1}$, Y. Pehlivan ${ }^{1}$, G. T. Jones ${ }^{1}$, I. Van der Horst-Bruinsma ${ }^{1}$, L. Midtbøll Ørnbjerg ${ }^{1}$, M. Ǿstergaard ${ }^{1}$, M. L. Hetland ${ }^{1} .{ }^{1}$ EuroSpA Research Collaboration Network, Copenhagen, Denmark

Background: There is a lack of real-life evidence on secukinumab effectiveness in psoriatic arthritis (PsA) patients. 
Objectives: To assess the real-life 6- and 12-month secukinumab retention rates and proportions of patients in remission/low disease activity (LDA) overall, and by prior biologic disease-modifying anti-rheumatic drug (bDMARD)/targeted synthetic (ts)DMARD use.

Methods: Data from PsA patients treated with secukinumab in routine care from 13 countries in the European Spondyloarthritis (EuroSpA) Research Collaboration Network were pooled. Patients started secukinumab $\geq 12$ months before date of datacut. Crude and LUNDEX adjusted (crude value adjusted for drug retention) 28-joint Disease Activity index for PSoriatic Arthritis (DAPSA28) and 28-joint Disease Activity Score with CRP (DAS28CRP) remission and LDA rates were calculated. Group comparisons between b/tsDMARD naïve, 1 prior and $\geq 2$ prior b/tsDMARD users were done with ANOVA, Kruskal-Wallis, Chi-square or Kaplan-Meier analyses with log-rank test, as appropriate.

Results: A total of 1543 PsA patients were included (Table 1). b/tsDMARD naïve patients had shorter time since diagnosis, higher baseline disease activity, a higher proportion were men and a higher proportion achieved remission. Overall 6/12-month secukinumab retention rates were $86 \% / 74 \%$ and significantly higher in b/tsDMARD naïve patients at 12, but not 6 months (Table 2, Figure). Overall, crude 6- and 12-month DAPSA28 $\leq 4 / \mathrm{DAS} 28 \mathrm{CRP}<2.6$ were achieved by $13 \% / 34 \%$ and $11 \% / 39 \%$ of the patients, respectively.

Table 1.

\begin{tabular}{|c|c|c|c|c|c|}
\hline & $\begin{array}{c}\text { All patients } \\
(n=1543)\end{array}$ & $\begin{array}{c}\text { b/tsDMARD } \\
\text { naïve }(n=287)\end{array}$ & $\begin{array}{l}1 \text { prior b/tsD- } \\
\text { MARD }(n=333)\end{array}$ & $\begin{array}{c}\geq 2 \text { prior b/ } \\
\text { tsDMARDs } \\
(n=923)\end{array}$ & $p^{*}$ \\
\hline $\begin{array}{l}\text { Age (years), mean } \\
\text { (SD) }\end{array}$ & $52(11)$ & 49 (12.3) & $51(11)$ & $53(11)$ & $<0.001$ \\
\hline Male, $\%$ & $42 \%$ & $49 \%$ & $46 \%$ & $39 \%$ & 0.003 \\
\hline $\begin{array}{l}\text { Years since diag- } \\
\text { nosis, mean } \\
\text { (SD) }\end{array}$ & $9(8)$ & $7(8)$ & $8(7)$ & $10(8)$ & $<0.001$ \\
\hline $\begin{array}{l}\text { Current smokers, } \\
\%\end{array}$ & $19 \%$ & $21 \%$ & $22 \%$ & $18 \%$ & 0.23 \\
\hline $\begin{array}{l}\text { CRP }(\mathrm{mg} / \mathrm{L}) \\
\text { median (IQR) }\end{array}$ & $5(2-12)$ & $7(2-19)$ & $4(2-8)$ & $5(2-11)$ & $<0.001$ \\
\hline $\begin{array}{l}\text { DAPSA28, median } \\
\text { (IQR) }\end{array}$ & $26(18-37)$ & $28(19-38)$ & $22(13-32)$ & $27(19-38)$ & $<0.001$ \\
\hline $\begin{array}{l}\text { DAS28CRP, } \\
\text { median (IQR) }\end{array}$ & $4.2(3.3-5.0)$ & $4.4(3.5-5.2)$ & $3.8(2.6-4.5)$ & $4.2(3.4-5.0)$ & $<0.001$ \\
\hline
\end{tabular}

${ }^{*}$ Comparisons across number of prior b/tsDMARD were done with ANOVA, Kruskal-Wallis or Chi-square test, as appropriate

Table 2.

\begin{tabular}{|c|c|c|c|c|c|c|}
\hline & Months & $\begin{array}{c}\text { All } \\
\text { patients } \\
(n=1543)\end{array}$ & $\begin{array}{c}\text { b/tsDMARD } \\
\text { naïve } \\
(n=287)\end{array}$ & $\begin{array}{c}1 \text { prior b/ } \\
\text { tsDMARD } \\
(\mathrm{n}=333)\end{array}$ & $\begin{array}{c}\geq 2 \text { prior b/ } \\
\text { tsDMARDs } \\
(n=923)\end{array}$ & $p^{*}$ \\
\hline \multirow{2}{*}{$\begin{array}{l}\text { Secukinumab } \\
\text { retention rate, \% } \\
(95 \% \mathrm{Cl})\end{array}$} & 6 & $\begin{array}{c}86 \% \\
(84-87 \%)\end{array}$ & $\begin{array}{c}89 \% \\
(86-93 \%)\end{array}$ & $85 \%(81-89 \%)$ & $85 \%(82-87 \%)$ & 0.11 \\
\hline & 12 & $\begin{array}{c}74 \% \\
(72-76 \%)\end{array}$ & $\begin{array}{c}81 \% \\
(76-86 \%)\end{array}$ & $76 \%(71-80 \%)$ & $72 \%(69-75 \%)$ & 0.006 \\
\hline \multicolumn{7}{|l|}{ DAPSA28 $\leq 4$} \\
\hline Crude & 6 & $13 \%$ & $25 \%$ & $11 \%$ & $11 \%$ & $<0.001$ \\
\hline LUNDEX & & $11 \%$ & $22 \%$ & $9 \%$ & $9 \%$ & $<0.001$ \\
\hline Crude & 12 & $11 \%$ & $22 \%$ & $11 \%$ & $8 \%$ & $<0.001$ \\
\hline LUNDEX & & $7 \%$ & $17 \%$ & $7 \%$ & $5 \%$ & 0.001 \\
\hline \multicolumn{7}{|l|}{ DAS28CRP $<2.6$} \\
\hline Crude & 6 & $34 \%$ & $51 \%$ & $33 \%$ & $30 \%$ & $<0.001$ \\
\hline LUNDEX & & $29 \%$ & $45 \%$ & $27 \%$ & $24 \%$ & $<0.001$ \\
\hline Crude & 12 & $39 \%$ & $55 \%$ & $41 \%$ & $34 \%$ & $<0.001$ \\
\hline LUNDEX & & $26 \%$ & $41 \%$ & $27 \%$ & $21 \%$ & $<0.001$ \\
\hline \multicolumn{7}{|l|}{ DAPSA28 $>4$ and } \\
\hline \multicolumn{7}{|l|}{$\leq 14$} \\
\hline Crude & 6 & $33 \%$ & $42 \%$ & $32 \%$ & $30 \%$ & 0.04 \\
\hline LUNDEX & & $27 \%$ & $37 \%$ & $27 \%$ & $25 \%$ & 0.02 \\
\hline Crude & 12 & $35 \%$ & $48 \%$ & $36 \%$ & $32 \%$ & 0.009 \\
\hline LUNDEX & & $24 \%$ & $36 \%$ & $24 \%$ & $20 \%$ & 0.004 \\
\hline \multicolumn{7}{|l|}{ DAS28CRP $\leq 3.2$} \\
\hline Crude & 6 & $52 \%$ & $69 \%$ & $53 \%$ & $47 \%$ & $<0.001$ \\
\hline LUNDEX & & $43 \%$ & $61 \%$ & $45 \%$ & $38 \%$ & $<0.001$ \\
\hline Crude & 12 & $55 \%$ & $72 \%$ & $55 \%$ & $50 \%$ & $<0.001$ \\
\hline LUNDEX & & $37 \%$ & $54 \%$ & $37 \%$ & $32 \%$ & $<0.001$ \\
\hline
\end{tabular}

${ }^{*}$ Comparisons across number of prior b/tsDMARDs were done with Kaplan-Meier with logrank test or Chi-Square test, as appropriate

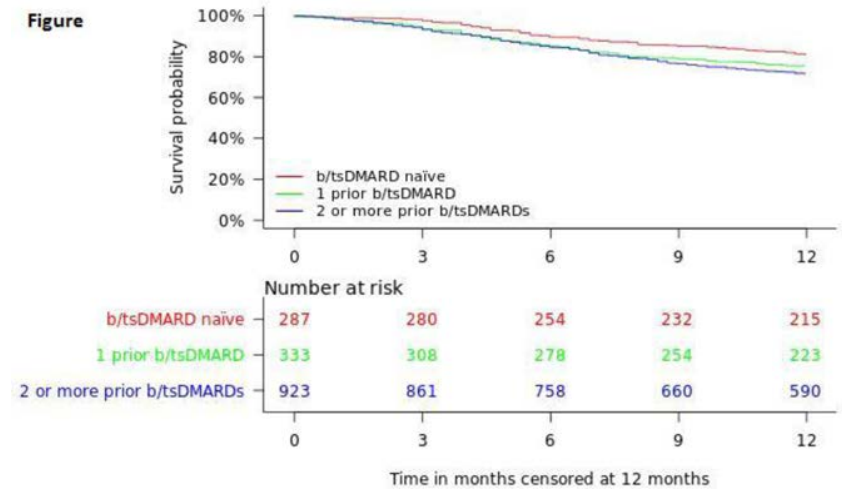

Conclusion: In this real-life study of 1543 patients with PsA in 13 European countries 12-month secukinumab retention was high, and significantly higher for b/tsDMARD naïve patients. Overall, a higher proportion of bionaïve than previous b/tsDMARD users achieved remission, regardless of remission criteria. Acknowledgments: Novartis and IQVIA for supporting the EuroSpA RCN Disclosure of Interests: Brigitte Michelsen Grant/research support from: Research support from Novartis, Consultant of: Consulting fees Novartis, Stylianos Georgiadis Grant/research support from: Novartis, Daniela Di Giuseppe: None declared, Anne Gitte Loft Grant/research support from: Novartis, Consultant of: AbbVie, MSD, Novartis, Pfizer and UCB, Speakers bureau: AbbVie, MSD, Novartis, Pfizer and UCB, Michael Nissen Grant/research support from: Abbvie, Consultant of: Novartis, Lilly, Abbvie, Celgene and Pfizer, Speakers bureau: Novartis, Lilly, Abbvie, Celgene and Pfizer, Florenzo lannone Consultant of: Speaker and consulting fees from AbbVie, Eli Lilly, Novartis, Pfizer, Roche, Sanofi, UCB, MSD, Speakers bureau: Speaker and consulting fees from AbbVie, Eli Lilly, Novartis, Pfizer, Roche, Sanofi, UCB, MSD, Manuel Pombo-Suarez Consultant of: Janssen, Lilly, MSD and Sanofi., Speakers bureau: Janssen, Lilly, MSD and Sanofi., Heřman Mann: None declared, Ziga Rotar Consultant of: Speaker and consulting fees from Abbvie, Amgen, Biogen, Eli Lilly, Medis, MSD, Novartis, Pfizer, Roche, Sanofi., Speakers bureau: Speaker and consulting fees from Abbvie, Amgen, Biogen, Eli Lilly, Medis, MSD, Novartis, Pfizer, Roche, Sanofi., Kari Eklund Consultant of: Celgene, Lilly, Speakers bureau: Pfizer, Roche, Tore K. Kvien Grant/research support from: Received grants from Abbvie, Hospira/Pfizer, MSD and Roche (not relevant for this abstract)., Consultant of: Have received personal fees from Abbvie, Biogen, BMS, Celltrion, Eli Lily, Hospira/Pfizer, MSD, Novartis, Orion Pharma, Roche, Sandoz, UCB, Sanofi and Mylan (not relevant for this abstract)., Paid instructor for: Have received personal fees from Abbvie, Biogen, BMS, Celltrion, Eli Lily, Hospira/Pfizer, MSD, Novartis, Orion Pharma, Roche, Sandoz, UCB, Sanofi and Mylan (not relevant for this abstract)., Speakers bureau: Have received personal fees from Abbvie, Biogen, BMS, Celltrion, Eli Lily, Hospira/Pfizer, MSD, Novartis, Orion Pharma, Roche, Sandoz, UCB, Sanofi and Mylan (not relevant for this abstract)., Maria Jose Santos Speakers bureau: Novartis and Pfizer, Björn Gudbjornsson Speakers bureau: Novartis and Amgen, Catalin Codreanu Consultant of: Speaker and consulting fees from AbbVie, Accord Healthcare, Alfasigma, Egis, Eli Lilly, Ewopharma, Genesis, Mylan, Novartis, Pfizer, Roche, Sandoz, UCB, Speakers bureau: Speaker and consulting fees from AbbVie, Accord Healthcare, Alfasigma, Egis, Eli Lilly, Ewopharma, Genesis, Mylan, Novartis, Pfizer, Roche, Sandoz, UCB, Sema Yılmaz: None declared, Johan K Wallman Consultant of: AbbVie, Celgene, Eli Lilly, Novartis and UCB Pharma, Cecilie Heegaard Brahe Grant/research support from: Novartis, Burkhard Moeller: None declared, Ennio Giulio Favalli Consultant of: Consultant and/or speaker for BMS, Eli-Lilly, MSD, UCB, Pfizer, Sanofi-Genzyme, Novartis, and Abbvie, Speakers bureau: Consultant and/or speaker for BMS, Eli-Lilly, MSD, UCB, Pfizer, Sanofi-Genzyme, Novartis, and Abbvie, Carlos Sánchez-Piedra: None declared, Lucie Nekvindova: None declared, Matija Tomsic: None declared, Nina Trokovic: None declared, Eirik kristianslund: None declared, Helena Santos Speakers bureau: AbbVie, Eli-Lilly, Janssen, Pfizer, Novartis, Thorvardur Love: None declared, Ruxandra lonescu Consultant of: Consulting fees from Abbvie, Eli-Lilly, Novartis, Pfizer, Roche, Sandoz, Speakers bureau: Consulting and speaker fees from Abbvie, Eli-Lilly, Novartis, Pfizer, Roche, Sandoz, Yavuz Pehlivan: None declared, Gareth T. Jones Grant/research support from: Pfizer, AbbVie, UCB, Celgene and GSK., Irene van der Horst-Bruinsma Grant/research support from: AbbVie, Novartis, Eli Lilly, Bristol-Myers Squibb, MSD, Pfizer, UCB Pharma, Consultant of: AbbVie, Novartis, Eli Lilly, Bristol-Myers Squibb, MSD, Pfizer, UCB Pharma, Lykke Midtbøll Ørnbjerg Grant/research support from: Novartis, Mikkel Ǿstergaard Grant/research support from: AbbVie, Bristol-Myers Squibb, Celgene, Merck, and Novartis, Consultant of: AbbVie, Bristol-Myers Squibb, Boehringer Ingelheim, Celgene, Eli Lilly, Hospira, Janssen, 
Merck, Novartis, Novo Nordisk, Orion, Pfizer, Regeneron, Roche, Sandoz, Sanofi, and UCB, Speakers bureau: AbbVie, Bristol-Myers Squibb, Boehringer Ingelheim, Celgene, Eli Lilly, Hospira, Janssen, Merck, Novartis, Novo Nordisk, Orion, Pfizer, Regeneron, Roche, Sandoz, Sanofi, and UCB, Merete L. Hetland Grant/research support from: BMS, MSD, AbbVie, Roche, Novartis, Biogen and Pfizer, Consultant of: Eli Lilly, Speakers bureau: Orion Pharma, Biogen, Pfizer, CellTrion, Merck and Samsung Bioepis

DOI: 10.1136/annrheumdis-2020-eular.1413

\section{\begin{tabular}{|l|l}
\hline SAT0431 PROPORTIONS OF PATIENTS ACHIEVING A MINIMAL \\
\hline
\end{tabular} DISEASE ACTIVITY STATE UPON TREATMENT WITH TILDRAKIZUMAB IN A PSORIATIC ARTHRITIS PHASE 2B STUDY}

$\underline{\text { P. Nash }}{ }^{1}$, M. E. Luggen², L. Espinoza ${ }^{3}$, F. J. García Fructuoso ${ }^{4}$, R. C. Chou ${ }^{5}$, A. M. Mendelsohn ${ }^{6}$, S. Rozzo ${ }^{6}$, I. Mcinnes ${ }^{7} .{ }^{1}$ School of Medicine Griffith University, Brisbane, Australia; ${ }^{2}$ Cincinnati Rheumatic Disease Study Group, Inc, and Univ of Cincinnati College of Medicine, Cincinnati, United States of America; ${ }^{3}$ LSU Health Sciences Center, New Orleans, United States of America; ${ }^{4}$ Hospital CIMA Sanitas, Barcelona, Spain; ${ }^{5}$ Univ at Buffalo School of Medicine and Biomedical Sciences, Buffalo, United States of America; ${ }^{6}$ Sun Pharmaceutical Industries, Inc, Princeton, United States of America; ${ }^{7}$ Univ of Glasgow, Glasgow, United Kingdom

Background: Tildrakizumab (TIL) is a high-affinity anti-interleukin-23p19 monoclonal antibody approved in the US, EU, and Australia to treat moderate to severe plaque psoriasis. A randomised, double-blind, placebo-controlled, multiple-dose, phase $2 \mathrm{~b}$ study evaluating the efficacy and safety of TIL was recently completed (NCT02980692).

Objectives: To characterise and evaluate the rate of minimal disease activity (MDA) up to week (W)52 from the phase $2 b$ study.

Methods: Patients $(\mathrm{pts}) \geq 18$ years old with active psoriatic arthritis $(\mathrm{PsA})^{2}$ and $\geq 3$ tender and $\geq 3$ swollen joints were randomised 1:1:1:1:1 to receive TIL $200 \mathrm{mg}$ every 4 weeks (Q4W) to W52, TIL $200 \mathrm{mg} \mathrm{Q12W}$ to W52, TIL $100 \mathrm{mg}$ Q12W to W52, TIL $20 \mathrm{mg} \mathrm{Q12W}$ to W24 $\rightarrow$ TIL $200 \mathrm{mg}$ Q12W to W52, or placebo (PBO) Q4W to W24 $\rightarrow$ TIL $200 \mathrm{mg}$ Q12W to W52. MDA was assessed throughout the study; an MDA response was achieved when 5 of 7 criteria were met. ${ }^{3}$ Safety was assessed throughout the study and included treatment-emergent adverse event (TEAE) monitoring.

Results: Of 500 pts screened, 391 were randomised and received $\geq 1$ dose of study drug. At baseline (BL), mean age was 48.8 years, $55 \%$ were female, $97 \%$ were White, mean body mass index was $29.7 \mathrm{~kg} / \mathrm{m}^{2}$, and pts had PsA for a median (range) of $4.4(0-42.8)$ years since diagnosis. Baseline disease characteristics related to MDA varied little between study arms (Table).

By W24, MDA state was achieved in significantly more pts receiving TIL vs PBO (24\%-39\% vs $7 \%$; $p<0.02$ for all groups); the proportion further increased with continued TIL treatment to W52 (45\%-64\%), including pts who switched from PBO to TIL (47\%) (Figure).

Among the overall pt population from $\mathrm{BL} \rightarrow \mathrm{W} 24 / \mathrm{W} 25 \rightarrow \mathrm{W} 52,50.4 \% / 39.9 \%$ and $2.3 \% / 1.0 \%$ experienced a TEAE and serious TEAE, respectively. From $B L \rightarrow W 24$, 1 serious infection (chronic tonsillitis) was reported for TIL $20 \mathrm{mg} \rightarrow 200 \mathrm{mg} \mathrm{Q12W}$ arm. From W25 $\rightarrow$ W52, there was 1 malignancy (TIL 20 $\rightarrow 200$ mg Q12W). There were no reports of candidiasis, uveitis, inflammatory bowel disease, major adverse cardiac events, or deaths from $\mathrm{BL} \rightarrow \mathrm{W} 24$ or W25 $\rightarrow \mathrm{W} 52$.

Table. Baseline disease characteristics related to minimal disease activity

\begin{tabular}{|c|c|c|c|c|c|}
\hline & $\begin{array}{c}\text { TIL 200mg } \\
\begin{array}{c}\text { Q4W } \\
n=78\end{array}\end{array}$ & $\begin{array}{c}\text { TIL 200mg } \\
\begin{array}{c}\text { Q12W } \\
n=79\end{array}\end{array}$ & $\begin{array}{c}\text { TIL 100mg } \\
\begin{array}{c}\text { Q12W } \\
n=77\end{array}\end{array}$ & $\begin{array}{c}\text { TIL } 20 \rightarrow 200 \mathrm{mg} \\
\text { Q12W } \\
n=78\end{array}$ & $\begin{array}{c}\mathrm{PBO} \rightarrow \text { TIL } \\
200 \mathrm{mg} \mathrm{Q12W} \\
\mathrm{n}=79\end{array}$ \\
\hline Swollen joint count & 10.4 & 10.0 & 11.0 & 9.4 & 11.8 \\
\hline Tender joint count & 16.6 & 19.5 & 21.3 & 19.0 & 19.7 \\
\hline Patient GADA score & 57.8 & 61.1 & 60.3 & 61.9 & 65.2 \\
\hline $\begin{array}{l}\text { Patient pain } \\
\text { assessment }\end{array}$ & 55.4 & 59.6 & 59.2 & 60.9 & 64.2 \\
\hline Enthesitis (LEI) score ${ }^{*}$ & 1.9 & 1.5 & 2.2 & 2.2 & 1.5 \\
\hline $\mathrm{PASI}^{\dagger}$ & 7.6 & 6.2 & 8.8 & 6.6 & 5.0 \\
\hline HAQ-DI score & 1.0 & 1.0 & 1.0 & 1.1 & 1.2 \\
\hline
\end{tabular}

Data are reported as mean.

*Total patients analysed $(n)=76,79,76,78,78$, respectively.

${ }^{\dagger}$ Total patients analysed $(n)=75,79,76,75,75$, respectively.

GADA, global assessment of disease activity; HAQ-DI, Health Assessment Questionnaire Disability Index; LEI, Leeds Enthesitis Index; PASI, Psoriasis Area and Severity Index; PBO, placebo; Q4W, every 4 hours; Q12W, every 12 hours; TIL, tildrakizumab.
Figure. Minimal disease activity response rates from baseline to week 52 in PSA patients across treatments and time points

$$
\begin{aligned}
& \text { † TIL } 200 \mathrm{mg} \mathrm{Q4W} \text { ₹ TIL } 200 \mathrm{mg} \text { Q12W }- \text { TIL } 100 \mathrm{mg} \text { Q12W } \\
& =\text { TIL } 20 \rightarrow 200 \mathrm{mg} \text { Q12W } \theta \text { Placebo } \rightarrow \text { TIL } 200 \mathrm{mg} \mathrm{Q12W}
\end{aligned}
$$

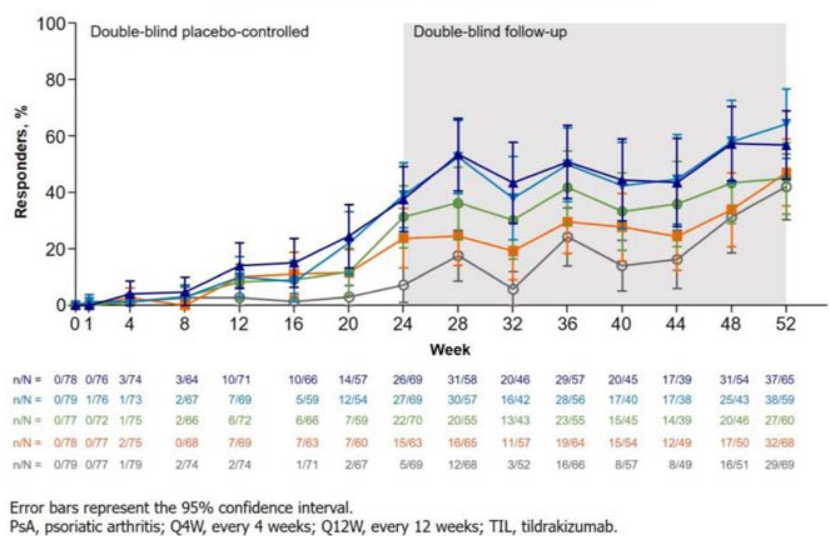

Conclusion: TIL produced clinically meaningful improvement in pts with PsA, resulting in a large proportion of pts achieving MDA by W52, and was well tolerated through W52.

References:

[1] Reich, et al. Lancet 2017;390:276-88.

[2] Taylor, et al. Arthritis Rheum 2006;54:2665-73.

[3] Coates, et al. Ann Rheum Dis 2010;69:48-53.

Disclosure of Interests: Peter Nash Grant/research support from: AbbVie, Bristol-Myers Squibb, Celgene, Eli Lilly and Company, Gilead, Janssen, MSD, Novartis, Pfizer Inc, Roche, Sanofi, UCB, Consultant of: AbbVie, Bristol-Myers Squibb, Celgene, Eli Lilly, Gilead, Janssen, MSD, Novartis, Pfizer Inc, Roche, Sanofi, UCB, Speakers bureau: AbbVie, Bristol-Myers Squibb, Celgene, Eli Lilly, Gilead, Janssen, MSD, Novartis, Pfizer Inc, Roche, Sanofi, UCB, Michael E Luggen Grant/research support from: AbbVie; Amgen; Eli Lilly; Genentech; Nichi-Iko; Novartis; Pfizer; R-Pharm; and Sun Pharmaceutical Industries, Inc., Consultant of: AbbVie; Amgen; Eli Lilly; Genentech; Nichi-lko; Novartis; Pfizer; R-Pharm; and Sun Pharmaceutical Industries, Inc., Speakers bureau: AbbVie; Amgen; Eli Lilly; Genentech; Nichi-Iko; Novartis; Pfizer; R-Pharm; and Sun Pharmaceutical Industries, Inc., Luis Espinoza: None declared, Ferran J García Fructuoso Grant/research support from: AbbVie, Eli Lilly, Gedeon Richter, Medlmmune, Nichi-Iko, Pfizer, Sanofi-Aventis, Takeda, and UCB, Consultant of: AbbVie, Eli Lilly, Gedeon Richter, Medlmmune, Nichi-Iko, Pfizer, Sanofi-Aventis, Takeda, and UCB, Speakers bureau: AbbVie, Eli Lilly, Gedeon Richter, Medlmmune, Nichi-Iko, Pfizer, Sanofi-Aventis, Takeda, and UCB, Richard C Chou Consultant of: Sun Pharmaceutical Industries, Inc, Alan M Mendelsohn Shareholder of: Johnson and Johnson, Employee of: Sun Pharmaceutical Industries, Inc, Stephen Rozzo Employee of: Sun Pharmaceutical Industries, Inc, lain McInnes Grant/research support from: Bristol-Myers Squibb, Celgene, Eli Lilly and Company, Janssen, and UCB, Consultant of: AbbVie, Bristol-Myers Squibb, Celgene, Eli Lilly and Company, Gilead, Janssen, Novartis, Pfizer, and UCB DOI: 10.1136/annrheumdis-2020-eular.3947

\section{SAT0432 \\ EFFECT OF SEX ON DISEASE CHARACTERISTICS AND DISEASE IMPACT IN PATIENTS WITH PSORIATIC ARTHRITIS (PSA): INSIGHTS FROM THE REAL- WORLD, OBSERVATIONAL MULTINATIONAL PSABIO COHORT}

M. T. Nurmohamed ${ }^{1}$, I. Van der Horst-Bruinsma ${ }^{2}$, A. W. Van Kuijk ${ }^{1}$, S. Siebert ${ }^{3}$ P. Bergmans ${ }^{4}$, K. De Vlam ${ }^{5}$, E. Gremese ${ }^{6}$, B. Joven-lbáñez ${ }^{7}$, T. Korotaeva ${ }^{8}$, W. Noel ${ }^{9}$, P. Sfikakis ${ }^{10}$, E. Theander ${ }^{11}$, J. S. Smolen ${ }^{12}$, L. Gossec ${ }^{13} .{ }^{1}$ VU University Medical Centre \& Reade, Amsterdam, Netherlands; ${ }^{2}$ UMC, Location VUmc, Amsterdam, Netherlands; ${ }^{3}$ University of Glasgow, Glasgow, United Kingdom; ${ }^{4}$ Janssen, Breda, Netherlands; ${ }^{5}$ University Hospitals, Leuven, Belgium; ${ }^{6}$ Catholic University of the Sacred Heart, Fondazione Policlinico Gemelli - IRCCS, Rome, Italy; ${ }^{7}$ Hospital 12 de Octubre, Madrid, Spain; ${ }^{8}$ Nasonova Research Institute of Rheumatology, Moscow, Russian Federation; ${ }^{9}$ Janssen, Brussels, Belgium; ${ }^{10}$ University of Athens Medical School, Athens, Greece; ${ }^{11}$ Janssen, Solna, Sweden; ${ }^{12}$ Medical University of Vienna, Vienna, Austria; ${ }^{13}$ Sorbonne University, Paris, France 